\title{
The use of brewer's spent grains in the cultivation of some fungal isolates
}

\author{
Olu. Malomo ${ }^{2, *}$, Daniels A. O. ${ }^{1}$, O. Olajiga ${ }^{1}$, Femi- Ola T. O. ${ }^{1}$, Alamu A.E ${ }^{2 .}$ \\ ${ }^{1}$ Department of Microbiology, University of Ado-Ekiti, Ado Ekiti, Ekiti State. Nigeria \\ ${ }^{2}$ College of Food Sciences, Bells University of Technology,Ota, Nigeria
}

\section{Email address:}

oludaremalomo1951@yahoo.com (Olu. Malomo)

\section{To cite this article:}

Olu. Malomo, Daniels A. O., O. Olajiga, Femi- Ola T. O. Alamu A.E.. The Use of Brewer'S Spent Grains in the Cultivation of Some Fungal Isolates, International Journal of Nutrition and Food Sciences. Vol. 2, No. 1, 2013, pp. 5-9. doi: 10.11648/j.ijnfs.20130201.12

\begin{abstract}
The use of brewery spent grains in the cultivation of some fungal isolates was investigated. Five fungal isolates namely: Rhizopus stolonifer, Rhizopus oligosporus, Mucor racemosus, Aspergillus niger and Mucor rouxii were selected. The growth and performance of these micro organisms were observed on substrate formulated from spent grains, while commercial yeast extract was used as a control of the experiment. Dry biomass were obtained were obtained using standard procedures and pellet sizes were determined by diameter measurement. The isolates showed best growth on substrate containing spent grains and commercial yeast powder. Aspergillus niger showed the highest extension growth of $380 \mathrm{~mm}$ at $144 \mathrm{hrs}$ in $0.9 \% \mathrm{w} / \mathrm{v}$ of spent grains and yeast powder combination in the ratio 1: 1 . The biomass production by each micro organisms peaked at the 90th hour.
\end{abstract}

Keywords: Fungal Isolates, Environmental Pollution, Brewers Spent Grain, Biomass

\section{Introduction}

With environmental pollution assuming an alarming rate in Nigeria, the disposal or further utilization of industrial waste has become an important factor in the efficient operation of any plant. Waste utilization or disposal in food industries is a major problem in maintaining sanitation and avoiding pollution of land, air and water [1]. Food waste contains such high concentration of nutrients carbohydrate, protein, fats and minerals. Such that if the waste are not immediately disposed off or utilized, due to high concentration of nutrients, it may attract insects, rodents, spoilage organisms or even pathogenic organisms [2]

Raw materials in form of food industrial waste have been channeled towards the production of industrial, commercial and pharmaceutical products that include energy and fine biochemical products [3] One man's meat is another man's poison. The waste of a company is seemingly the raw materials of another. In actual sense, no waste is a waste. The waste generated by the beer industry is becoming complex, difficult to treat and expensive. Since it is economically feasible and possible to develop many economic and sellable products from brewing by- products, not only is disposal cost saved, it helps in prevention of environmental pollution, helps in Agriculture and also in human and animal nutrition.

Brewer spent grains (BSG) are the major by-product of the beer industry, representing around $85 \%$ of the total byproduct generated. BSG contains $17 \%$ cellulose, $28 \%$ non cellulosic polysaccharide and 38\% lignin [4].. BSG can serve as an adjunct in human nutrition due to its high protein content. They have also been used in feeding both ruminant and monogastric animals. It has also been shown to be an excellent media for microbial growth of yeasts and moulds [5]

Recently, attempts have been made to use BSG in biotechnological processes such as in the cultivation of mushrooms and Actinobacteria [5]. BSG is also used as supplements in baking and confectionaries up to $30 \%$ substitute [6]. BSG contains fiber, protein, lipid, ash, minerals, vitamins, and amino acids [7].Other uses of BSG include: energy production, brick components, charcoal, paper manufacture, biotechnological processes, as adsorbent and as additives or carrier in brewing and also as substrate for microorganism cultivation.

As a result of the usefulness of BSG, the need to prevent environmental pollution as well as the need to search for a cheaper and readily available medium for the cultivation of microorganisms, this work was carried out to look into the possibility of preparing medium from BSG for the cultiva- 
tion of some fungal isolates.

\section{Material and Methods}

\section{Collection and preparation of spent grains}

Brewer's spent grains ( Barley ) was collected from International Breweries Plc, Ilesa, Osun State, Nigeria. The wet spent grain was dried at room temperature to prevent enzyme denaturation. The dried spent grain was grounded into powder using the laboratory blender.

\section{Collection of test microorganisms}

Seven days old cultures of five different fungal isolates were selected after inoculating suitable media with dilute preparation of yoghurt[6][7]. The test organisms were identified as Rhizopus stolonifer, Rhizopus oligosporus, Mucor racemosus, Aspergillus niger and Mucor rouxii.

\section{Preparation of inocula}

Each isolate was transferred into $50 \mathrm{ml}$ distilled water in a test tube and shaken vigorously for 20 seconds, spore concentration of $10^{6}$ spores $/ \mathrm{ml}$ was inoculated.

\section{Media preparation}

Dried spent grain was ground into powder and particulate matters were removed. 60 grams of spent grain powder was steeped in $250 \mathrm{ml}$ of sterile distilled water for $48 \mathrm{hrs}$ and the supernatant was decanted to obtain extract. The basal medium contains the following in $\mathrm{g} / \mathrm{l}$ (NH4) NO3, 4.0; NH4Cl; 20.0, KH2PO4, 4.0; K2HPO4, 12.0; Na2SO4, 8.0; andMgSO4, 0.4 in 11 of distilled water.

Three different combinations of liquid media were prepared. The first contained a basal medium and spent grains in varied concentrations. The second contained basal medium and commercial yeast powder (Oxoid) also in varying concentrations. A third combination contained basal medium, spent grains, and yeast powder. All media preparations were sterilized at $121^{\circ} \mathrm{C}$ for 15 minutes. The media were solidified by using 15\% Agar no 3 (Oxoid) as the solidifying agent. The concentration of the spent grains and yeast powder varied in which $0.6 \% \mathrm{w} / \mathrm{v}$ of both the grains and yeast powder was used in one and $0.9 \% \mathrm{w} / \mathrm{v}$ was used in the other.

\section{Shake flask fermentation}

After preparation of the liquid media, $100 \mathrm{ml}$ portion of each combination were dispensed into $500 \mathrm{ml}$ shake flasks and $1 \mathrm{ml}$ each of homogenized fungal inoculums was introduced into the flask using sterile syringe. These were then fermented at $28.2^{\circ} \mathrm{C}$. Samples were collected at different intervals and analyzed for biomass production.

\section{Biomass determination}

Each sample was collected at different intervals of 48 , $72,96,120$ hours. The growth medium was pasteurized at $65{ }^{\circ} \mathrm{C}$ for 30 minutes and centrifuged at $3000 \mathrm{~g}$ in Baird and Tatlock auto bench centrifuge Mark IV (Denley, England). The supernatant was decanted and mycelial pellets washed free of the medium four times with sterilized but cooled distilled water. This was then passed through dried and preweighed Whatman No 1 filter paper. Biomass on filter pa- per was dried at $60 \pm 2{ }^{\circ} \mathrm{C}$ for $48 \mathrm{hrs}$ to a constant weight in a hot oven. This was allowed to cool and the final weight of the dry biomass was obtained and recorded.

\section{Solid media inoculation}

Fungal inocula were point inoculated at the centre of media plates and incubated at $30^{\circ} \mathrm{C}$.

Determination of growth extension.

The growth extension diameter $(\mathrm{mm})$ of the fungi was measured at different intervals of 48, 72, 96, 120 and 144 hours using a vernier caliper.

\section{Statistical analysis}

Statistical analyses of data obtained in this work were carried out using the one way ANOVA at $95 \%$ confidence limit of (SPSS 7.0).

\section{Results}

The extension growth measurements of the five fungal isolates inoculated on spent grain medium and commercial yeast powder medium are recorded in Table 1. Aspergillus niger showed the highest growth rate (Table 1) amongst all the cultivated microorganisms. The table also showed that the highest rate of growth of Aspergillus niger was observed on substrate combination of $0.9 \% \mathrm{w} / \mathrm{v}$ of spent grain and commercial yeast powder (SG-YP) with an extension growth of $380 \mathrm{~mm}$ at $144 \mathrm{hrs}$.Mucor racemosus and Mucor rouxii (Table 1) showed no growth on the solid media prepared throughout the period of observation. Furthermore, it was observed that Rhizopus stolonifer showed its best growth on substrate containing only spent grains as compared with other substrate combinations. Rhizopus oligosporus showed best growth on substrate containing spent grains and commercial yeast powder (Table 1)

Statistical analyses of the results obtained for the extension growth measurement of Rhizopus stolonifer, Rhizoopus oligosporus and Aspergillus niger showed that R. stolonifer and A.niger on the various substrate combination $(\mathrm{P} \leq 0.5)$, However, R. oligosporus showed similar growth rate on the various substrate combination on $0.6 \% \mathrm{w} / \mathrm{v}$ yeast powder and $0.9 \% \mathrm{w} / \mathrm{v}$ of spent grains and yeast powder. Statistical analyses of the result obtained for the biomass production showed that there are no significant differences $(\mathrm{P} \leq 0.5)$ in the biomass production for R. oligosporus, $M$. racemosus. A. niger, and $M$. rouxii in the various substrate combinations Rhizopus stolonifer, however, showed similar biomass production in increasing order in the following groups of substrate combinations; Group one --- $0.6 \% \mathrm{w} / \mathrm{v}$ spent grains, $0.6 \% \mathrm{w} / \mathrm{v}$ spent grains and yeast powder and $0.9 \% \mathrm{w} / \mathrm{v}$ spent grains. Group two---0.6\% w/v spent grains and yeast powder, $0.9 \% \mathrm{w} / \mathrm{v}$ spent grains and $0.6 \% \mathrm{w} / \mathrm{v}$ yeast powder. Group three--- $0.9 \% \mathrm{w} / \mathrm{v}$ spent grains, $0.6 \% \mathrm{w} / \mathrm{v}$ yeast powder and $0.9 \% \mathrm{w} / \mathrm{v}$ yeast powder. Statistical analyses showed that there are no significant differences $(\mathrm{P} \leq 0.5)$ in biomass production for Rhizopus oligosporus, Mucor racemosus, Aspegillus niger and $\mathrm{Mu}-$ cor rouxii in the various substrate combinations. However, 
Rhizopus stolonifer showed similar biomass production in increasing order in the combinations found in groups one, two and three.

The biomass cropped from the fungal isolated after the cultivation in the liquid media are shown in figure 1 . As can be observed from the graphical representations, all the fungal isolates demonstrated their best growth in $0.9 \% \mathrm{w} / \mathrm{v}$ yeast powder except Aspergillus niger which showed high- est growth in substrate combinations of spent grains and yeast powder. (Fig 1).Aspergillus niger had the overall highest growth and was at its best in $0.9 \% \mathrm{w} / \mathrm{v}$ of spent grains and yeast powder.

Mucor racemosus was at its best in $0.9 \% \mathrm{w} / \mathrm{v}$ of spent grains and commercial yeast powder. Generally, rate of increase in biomass production peaked at the 96th hour as shown in the graphical representation. (1 to 3 ).

Table 1. Extension growth ( $\mathrm{mm}$ ) and time taken for fungal growth using formulated media.

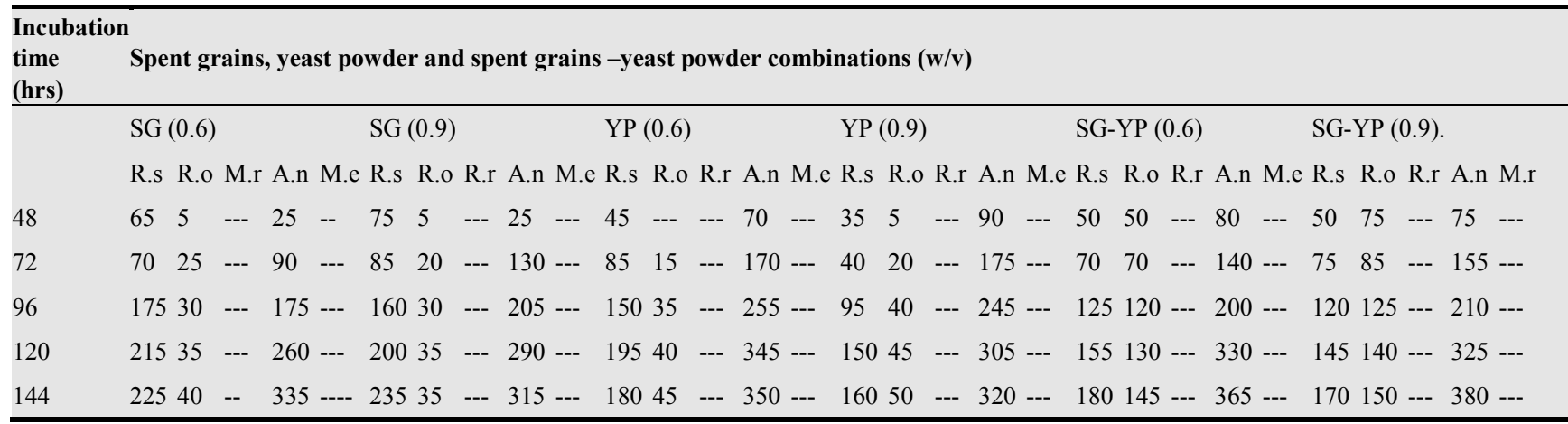

Legend ; SG----Spent grains, R.o---- Rhizopus oligosporus, YP---- Yeast powder, M.r---- Mucor racemosus., SG-YP----- Spent grain- Yeast powder combination, A.n----Aspergillus niger, R.s---- Rhizopus stolonifer, Me---- Mucor rouxii.

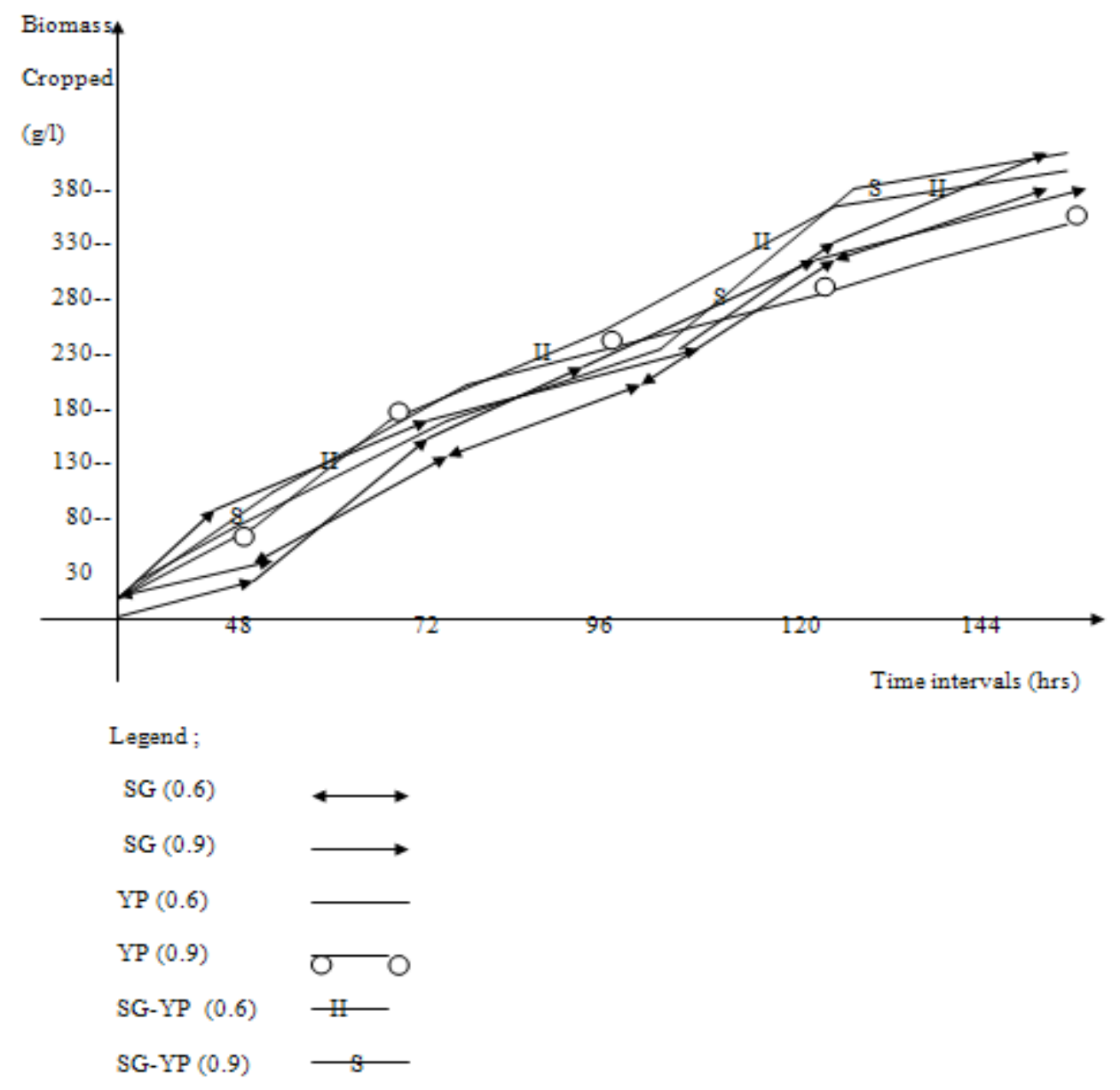

Figure 1. Plot of biomass cropped from Aspergillus niger at different time intervals. 

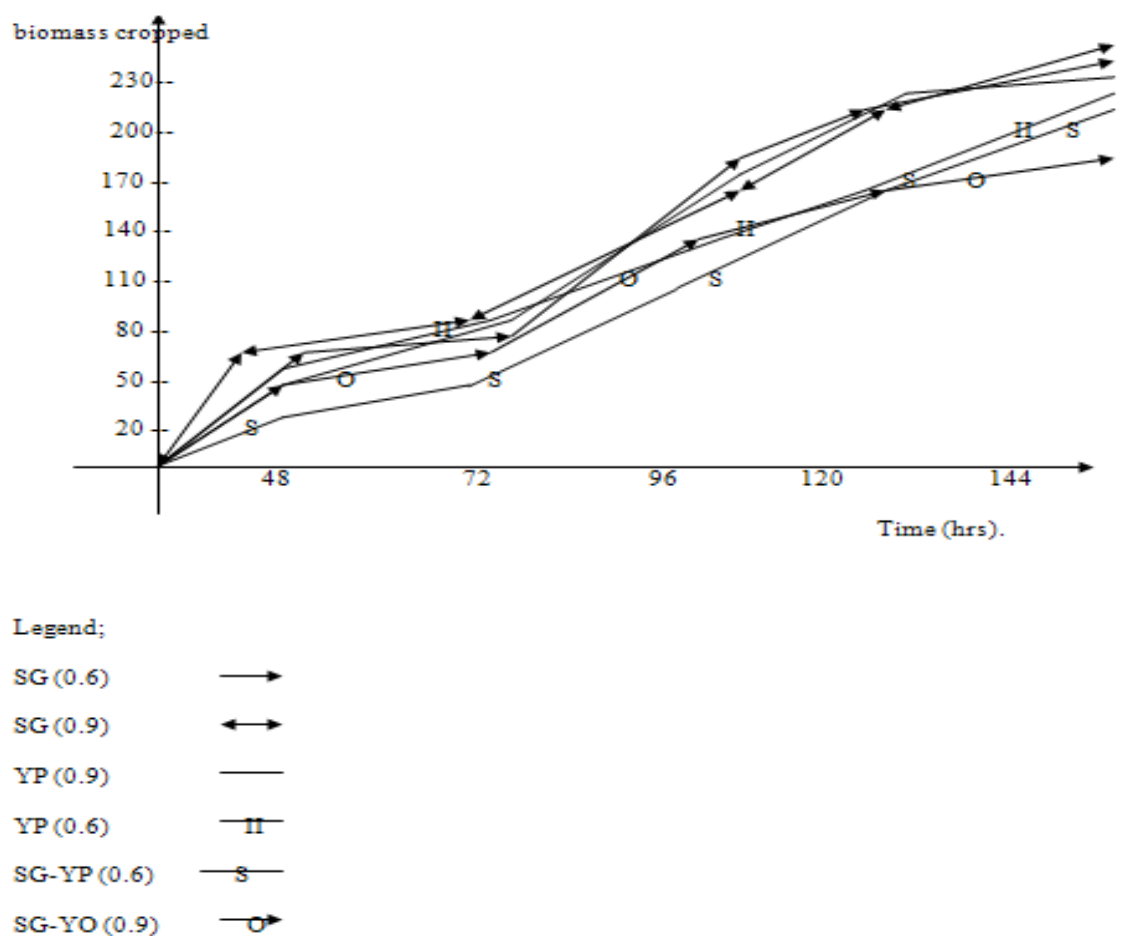

Figure 2. Plot of biomass cropped from Rhizopus stolonifer at different time intervals.

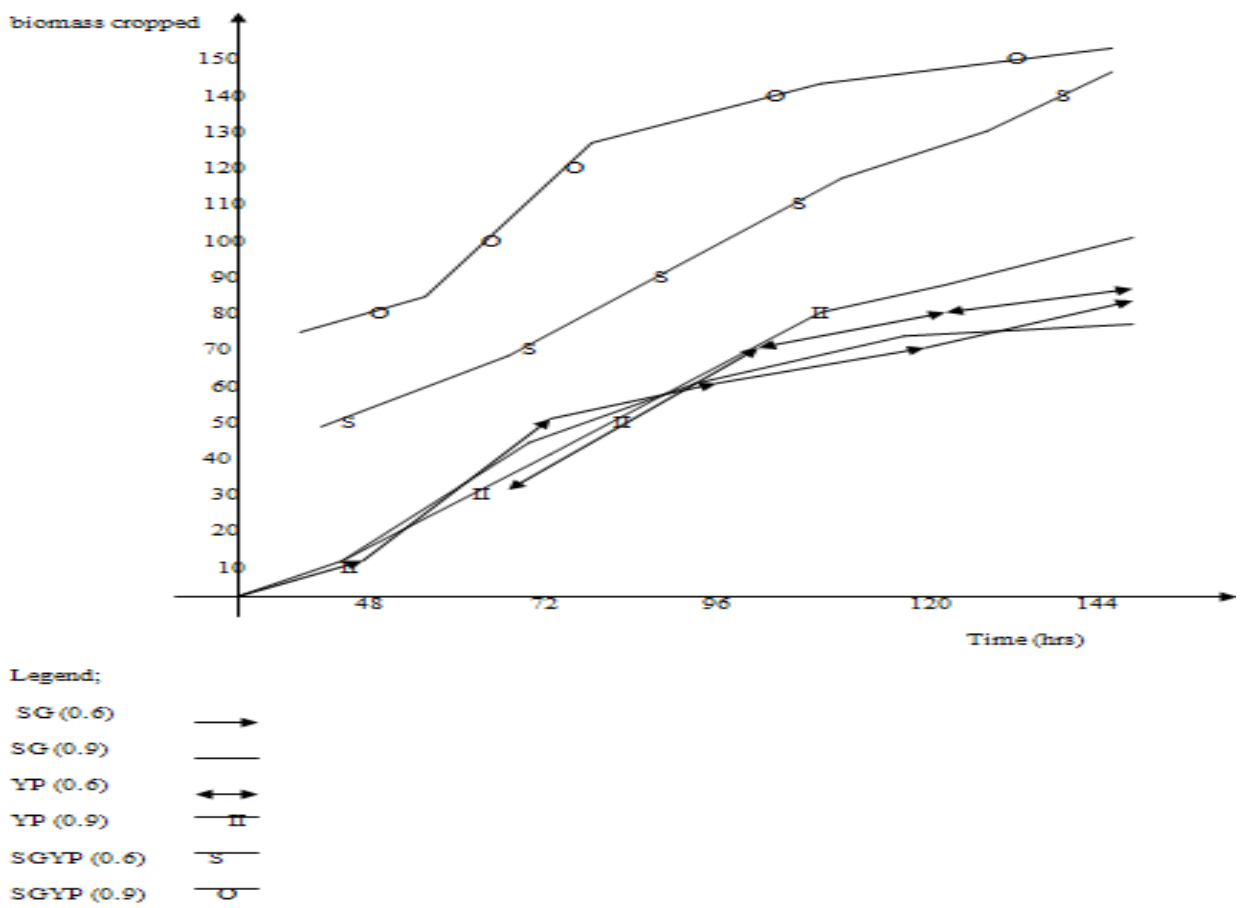

Figure 3. Plot of biomass cropped from Rhizopus oligosporus at different time intervals.

\section{Discussion}

Increasing efforts are being directed towards the reuse of agro-industrial by-products, from both economical and environmental point of view. Among the five fungal isolates used in this study, Aspergillus niger gave fastest growth measurement and had the highest dry biomass production in the substrate combinations of spent grains and yeast powder $(0.6 \% \mathrm{w} / \mathrm{v}$ SGYP.). This supports the work of Sangeeta et al.,[7], who observed a high amylolytic activity of Aspergillus niger while Mussato,[4] found that Aspergillus niger is superior to other species of Aspergillus and Rhizopus in amylase activity. These observations might explain the highest extension growth and biomass produced by Aspergillus niger as compared to other fungal isolate cultured.

The mean dry biomass harvested at the end of the $48 \mathrm{hr}$ 
fermentation of Aspergillus niger was $15.35+0.05$. Gregory et al., [1] observed in his work that fastest mycelia growth was observed in substrate containing $68 \%$ brewer's spent grains. Wang et al., [8], using Aspergillus niger grown in brewery spent grains liquor obtained a lower dry biomass of $13 \mathrm{~g} / \mathrm{l}$ of medium. Gregory et al.,[1] also discovered that a higher growth rate and biomass production resulted with the BSG agar than malt extract agar when used to culture Pleurotue ostreatus. Wang et al., [8], also obtained highest yield of enzymes on substrate containing $55 \%$ BSG. The difference in biomass reported might be due to the difference in substrate and fermentation time. Many authors have noted the importance of supplementation in increasing biomass as well as the protein content of biomass. However, the supplementation levels designate which enzyme are produced and in what amount [1]. In recent study, $\mathrm{Xu}$ et al,[9], established the amylase production capacity of Aspergillus oryzae on brewer's spent grain.

Common agricultural waste littering dump sites in many developing countries can serve as substrate for many biologically useful materials like amylases and aminoglucodases

\section{Conclusion}

BSG is a by-product that can be obtained from breweries. The use of BSG is still limited and as such the development of new techniques to use this agro-industrial byproduct is of great importance since spent grains are produced in large quantities throughout the year. Since spent grain is majorly composed of carbohydrate, more attention should be paid to its conversion to soluble and fermentable sugars.

According to our findings, BSG could be an appropriate substrate for the cultivation of fungal isolates especially Aspergillus niger. This study has also provided information on the suitability of BSG as substrate for the cultivation of fungal isolates. However, adaptation of different fungal strains for the growth on BSG in combination with other substrate could be looked into. Furthermore, the use of
BSG for enzyme production could also be explored preferably looking into BSG with different substrate supplementation rates.

\section{References}

[1] Gregory, A. S., Mirjan, P. Bojan, and F Pohleven (2008). The use of Brewery grains pleurotus

[2] ostreatus cultivation and enzyme production. New Biotechnology. Vol 00 number 00

[3] Miller, B. (1998). Fat of the land. New York waste. Social Research, 65, 111-150

[4] Mussato, S.I.,Roberto, I.C. (2005). Acid hydrolysis and fermentation of Brewer's spent grain to produce Xylitol. Journal of the Science of Food and Agriculture (in press).

[5] Mussato, S.I. (2006). Brewer's spent grains generation, characterization and potential application. Journal of Cereal Science.43,1-14.

[6] Nascimento, R.P., Coelho, R.R.R., Marques, S.., Alves, L.Girio, F.M., Bon, E.P.S., Amaral-Collaco, M.T (2002).Production and partial characterization of Xylase from Streptomyces spp strain AMT-3 isolated from Brazillian cerrado soil. Enzyme and Microbial Technology, 31, 549-555.

[7] Santo, M.,Jimenez, J.J, Batolome, B., Gomez-Cordoves, C. delNozal, M.J. (2003).Variability of Brewer's spent grains within a Brewery. Food Chemistry, 80, 17-21.

[8] Sangeeta, P.T., Ramesh, M.N., Prapulla, S.G (2004),Production of fructosyl transferase by Aspergillus oryzea CFR 202 in solid state fermentation using agricultural by- products. Applied Microbiology and Biotechnology, $65,530-537$.

[9] Wang, D.(2001). Biological efficiency and nutritional value of Pleurotus ostreatus cultivated on spent beer grain. Bioresource Technology,78,293-300.

[10] 9. Xu, H. Sun, L. Zhao, D. Zhang, B.Shi, Y. Wu, Y. (2008).Production of amylase by Aspergillus oryzae as 3951 in solid state fermentation using spent grains substrate. Journal of Science Food Agric,88 (3).529-535. 